\title{
Experiences of continuing medical education during COVID 19
}

\author{
Callum Robertson ${ }^{1}$, Grant Tong ${ }^{2}$, and Lew-Chin Chee ${ }^{2}$ \\ ${ }^{1}$ Queen Elizabeth University Hospital \\ ${ }^{2}$ Inverclyde Royal Hospital
}

July 7, 2020

\begin{abstract}
Abstract Journal clubs are a valued source of teaching for medical professionals. We launched a novel podcast delivered journal club utilising commonly used social media applications to drive asynchronous discussion around key topics. This design uses commonly accessible freeware and can be customised to suit the cohort of participants. We demonstrate changes of practice achieved through the journal club discussion, a sustained response of contributors, links to external papers and links to external media to augment the discussion. We are hoping to launch this intervention at our sister sites and assess over a longer time frame to demonstrate sustainability and validity of this teaching intervention.
\end{abstract}

\section{Introduction}

Journal clubs are a valued source of teaching but 'classic journal clubs' are being challenged during social distancing measures. Whilst I.T. solutions could overcome these challenges they can be expensive or limited by reduced user acceptability ${ }^{1}$. In addition, synchronous teaching can be difficult due to alternative working patterns which may limit participation and networking opportunities with video conferencing. Nevertheless, virtual journal clubs have been gaining popularity amongst doctors and medical students ${ }^{2}$. Twitter journal clubs have demonstrated asynchronous discussions to allow greater participation and wider networking opportunities but appear less relevant for local QI opportunities, as well as being hindered by its lack of popularity in comparison to other social media platforms ${ }^{3}$. We explored a style of asynchronous digital journal club, not previously reviewed in the literature, utilising common free social media platforms and aspects from cognitive learning theory.

\section{Methods}

Our design was a podcast facilitated journal club with asynchronous chat hosted in a cohort specific social media platform. Learning from previous studies, users have limited participation in a non-native platform ${ }^{2}$. Before starting the journal club, we utilised a survey to understand our demographic. The results of this survey of 7 consultants and 8 trainees from a busy DGH department, is shown in figure 1. This highlighted that all respondents used the WhatsApp social media tool, which we decided to host our discussion board on. Podcasts were made each month by a trainee who would pick a recently released paper from a preapproved topic and produce a 5-minute structured summary of the paper using the PICO format ${ }^{4}$. This design was to optimise cognitive load by removing extraneous information to best relay key messages ${ }^{5}$. These podcasts were produced using free software. Podcasts were uploaded at the start of each month and the host would moderate the chat as necessary to encourage discussion around key issues. At the end of the month a summary document would be provided for CPD purposes. After 3 months we launched a further SurveyMonkey and collated the metrics from the discussion board. This included a Likert scale question on satisfaction with the virtual journal club. A change of practice was defined as consensus agreement from the discussion board to change an aspect of regular care in response to the paper or supplementary media. 


\section{Results}

Survey results after the first 3 months of our virtual journal club are shown in figure $2(n=8)$. All 8 respondents rated the virtual journal club design $5 / 5$ on the Likert scale. The 7 consultants had posted at least once during this time frame. All trainees, with the exception of 2 , commented at least once in the 3 months. Podcast views suggested each topic was listened to greater than 15 times.

Discussion

Our model appears to be successful, achieving a high satisfaction rate as evident from the SurveyMonkey. In addition, as each podcast was listened to in excess of the number of participants, it would imply that everyone listened to it at least once. In addition, as a trainee led journal club it offers a sustainable and reproducible model. There is inert motivation to contribute in order to demonstrate CPD and this offers an opportunity to participate in a presentation without the time commitment of a 'classical journal club'

There appears to be some advantages of this model. It is cost neutral and can be adapted to suit the cohort of participants. The asynchronous design overcomes the challenges posed by clinical commitments. A time frame of one month per journal club allows participation around social circumstances to maximise networking. We appear to have a sustained response demonstrated by the metrics with a regular number of posters and posts. In addition, unlike some virtual journal clubs, this design requires minimal I.T. knowledge. Utilising applications familiar to the cohort may in part be responsible for the acceptability of this design.

Limitations of this study are the small sample size and short time frame of assessment. Notably our centre's classical journal club had been disbanded before the pandemic due to poor attendance so although this model has been initially successful, a longer time frame would be beneficial in establishing sustainability. We can assess the validity of this design using the Kirkpatrick model where we can argue the changes to practice documented are examples of result attainment ${ }^{6}$. It is our aim to test this model in a larger cohort to better assess its long-term sustainability and validity.

\section{References}

1. Hammond D, Alexander K, Rech M, et al. Professional Benefits of a Web-based Journal Club for Critical Care Residents and their mentors.American Journal of Pharmaceutical Education. 2019;83.

2. Dovi G. Empowering change with traditional or virtual journal clubs.Nursing Management. 2015;46(1):4650 .

3. Udani A, Moyse D, Peery C, Taekman J. Twitter-Augmented Journal Club: Educational Engagement and Experience So Far. A $\mathcal{E}$ A Case Reports. 2016;6:253-256.

4. Cooke A, Smith D, Booth A. Beyond PICO: The SPIDER Tool for Qualitative Evidence Synthesis. Quality Health Research.2012;22:1435-1443.

5. Sweller J. Cognitive load theory, learning difficulty, and instructional design. Learning and Instruction.1994;4(4):295-312.

6. Kirkpatrick DL. Evaluating training programs: The four levels.San Francisco: Berrett-Koehler; 1994. 
Last Read a Research Paper

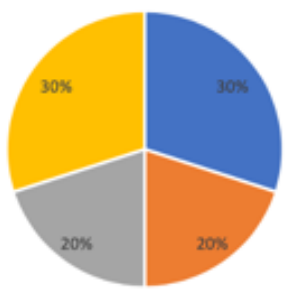

- Past Month $\quad=1-2$ Months $\approx 0$

- 2-6 Months ago $=>6$ months aeo

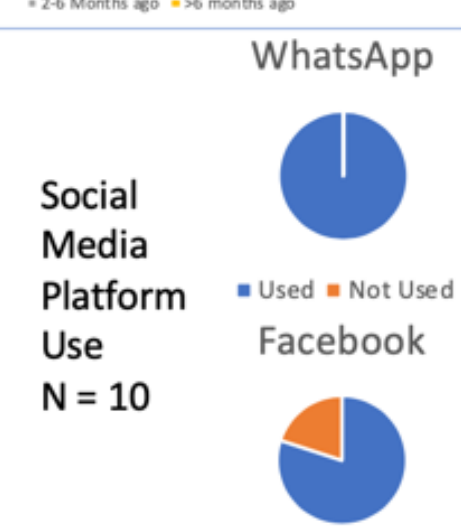

- Used = Not Used
Last Attended a Journal Club

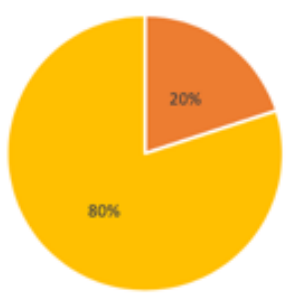

- $\angle 1$ Month $A_{g} \circ$ = 1.2 Months Ago

=2-6 Months Ago = >6 Months Ago

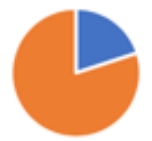

- Used = Not Used

Youtube

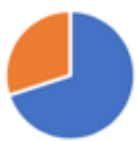

- Used not Used

\begin{tabular}{|c|c|c|c|c|c|}
\hline & $\begin{array}{l}\text { Number of posts } \\
\text { (not including host) }\end{array}$ & \multicolumn{2}{|c|}{$\begin{array}{l}\text { Attachments to } \\
\text { webinar/other } \\
\text { papers }\end{array}$} & Changes to practice & $\begin{array}{l}\text { Number of unique } \\
\text { posters }\end{array}$ \\
\hline Paper 1 & 33 & \multicolumn{2}{|l|}{5} & 3 & 8 \\
\hline Paper 2 & 27 & \multicolumn{2}{|l|}{10} & 3 & 9 \\
\hline Paper 3 & 29 & \multicolumn{2}{|l|}{8} & 1 & 7 \\
\hline \multicolumn{3}{|c|}{$\begin{array}{l}\text { Free Text Responses - baseline survey } \\
\text { - Excellent idea! } \\
\text { - I sometimes can't get out to regular teaching } \\
\text { and feel like I'm missing out, hopefully this } \\
\text { solves this } \\
\text { - Good idea! } \\
\text { - Excellent idea } \\
\text { - Woop Woop } \\
\text { - Brilliant idea } \\
\text { - The number of times you can't join into a } \\
\text { journal club because of shift patterns, It's a } \\
\text { great idea }\end{array}$} & \multicolumn{3}{|c|}{$\begin{array}{l}\text { Free Text Responses - After } 3 \text { months } \\
\text { - Great idea } \\
\text { - Sometimes struggled with time to post, good } \\
\text { that its on for so long } \\
\text { - Would be good to have a summary video chat } \\
\text { at the end, but at least everyone can } \\
\text { participate this way } \\
\text { - Like that it was a bit different } \\
\text { - Sometimes felt guilty for not posting but } \\
\text { people had already said what I wanted to say }\end{array}$} \\
\hline
\end{tabular}

